\title{
76. DEVIATION OF THE VERTEX OF THE VELOCITY ELLIPSE OF YOUNG STARS AND ITS CONNECTION WITH SPIRAL STRUCTURE
}

\author{
SIR R. WOOLLEY \\ Royal Greenwich Observatory, Hailsham, Sussex, England
}

\begin{abstract}
The deviation of the vertex of the velocity ellipse of stars of spectral class A is shown clearly in a diagram published by Strömberg (1946); no such clear deviation is shown by the velocity ellipses of stars of other spectral types in his diagrams. This deviation of the vertex or tilt of the velocity ellipse can have some connexion with spiral struct ure, and there seem to be two possibilities, (a) that the tilt is confined to young stars only, and has something to do with an initial condition of their formation; and (b) that the tilt is a manifestation of the nature of the attracting field in the neighbourhood of the sun, which is not central on account of the attractions of spiral arms.

The present note examines data taken from the velocities of nearby stars, and uses the classification of the late type main sequence stars put forward by Wilson, based on observations of the reversals in the $\mathrm{Ca}^{+} \mathrm{H}$ and $\mathrm{K}$ lines.

From this material it is concluded that the vertex deviation is confined to young stars, and that hypothesis (a) rather than hypothesis (b) is correct - or at least strongly indicated by available observations. The paper goes on to show that the deviation of the vertex can be explained by supposing that the stars were formed comparatively recently in a thin strip of the galaxy more or less at right angles to the direction of the galactic centre - in fact, in something like a spiral arm.
\end{abstract}

1. The velocities discussed in this note are constructed from trigonometrical parallaxes and proper motions, and from radial velocities. They are taken from Gliese's (1957) catalogue and its revision (Gliese, 1969), and from an extension of Gliese's catalogue which uses trigonometrical parallaxes down to 0."040 now being compiled at the Royal Greenwich Observatory. The transverse and radial velocities are solved along rectangular $(U, V, W)$ axes of which $U$ is directed towards the centre of the galaxy. $V$ is in the galactic plane in the direction in which rotation takes place and $W$ is perpendicular to the galactic plane and positive northwards. These velocities have been referred to an adopted standard of rest which has the velocities

$$
\begin{aligned}
& U=-10 \mathrm{~km} \mathrm{~s}^{-1} \\
& V=-10 \mathrm{~km} \mathrm{~s}^{-1} \\
& W=-7 \mathrm{~km} \mathrm{~s}^{-1}
\end{aligned}
$$

relative to the sun.

2. The vertex deviation may be examined quite readily by inspection of a simple plot of the $U$ and $V$. Three figures are shown. Figure 1 shows all the A stars in Gliese's catalogue (filled circles) and all the very young late type main sequence stars according to Wilson's criterion (open circles). (These are stars to which he assigns the $\mathrm{Ca}^{+}$emission index +3 . A paper discussing this criterion has been prepared by Wilson and Woolley.) The figures representing old stars are left separate to avoid crowding and Figure 2 shows the velocities of stars to which Wilson assigns zero (crosses) or negative 


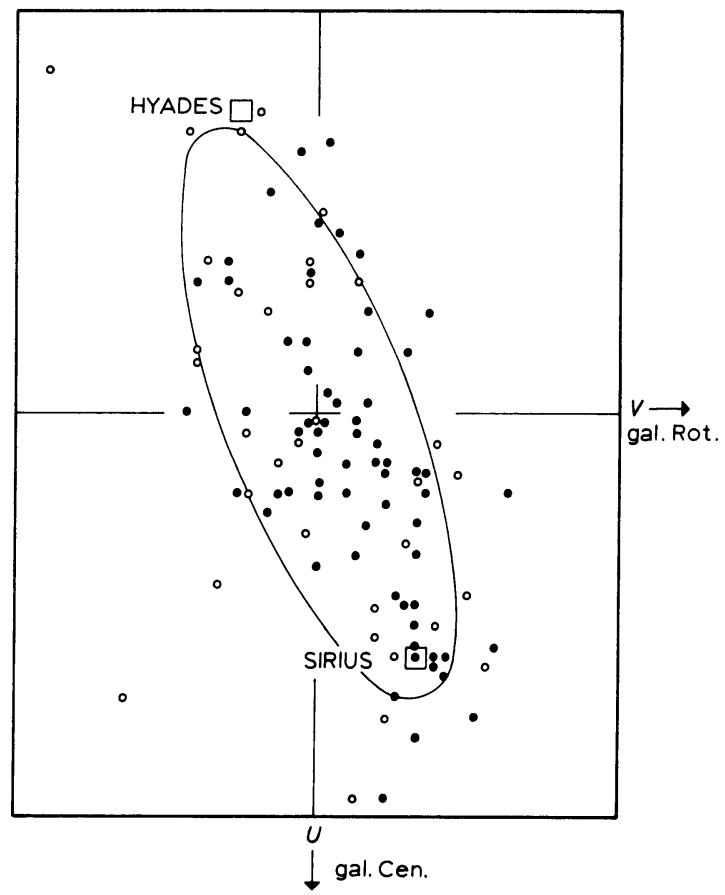

Fig. 1. Velocities of A-type stars in the solar neighbourhood (O) and of late type main sequence stars to which Wilson assigns $\mathrm{Ca}^{+}$emission intensities of +3 or greater $(\bigcirc)$.

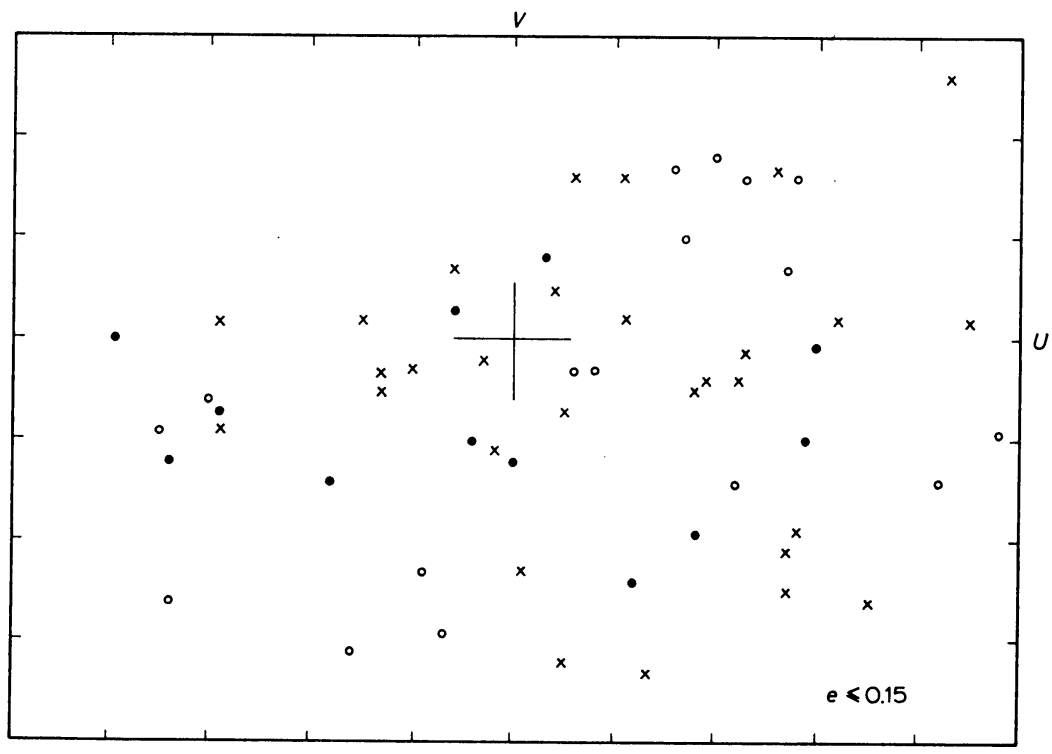

Fig. 2. Velocities of late type main sequence stars with $\mathrm{Ca}^{+}$emission zero or negative: Wilson No. zero $(\times)$, Wilson No. $-1,(\bigcirc)$, Wilson No. $-2,-3,-4(\bigcirc)$. 
calcium emission (open circles). Figure 3 shows the corresponding diagram for evolved stars in Gliese's catalogue, that is stars of MK luminosity classes II, III and IV and of spectral type later than F5, the different symbols representing different spectral types in this diagram.

In Figure 1 the vertex deviation is obvious and in both Figures 2 and 3 show little sign, if any, of it.

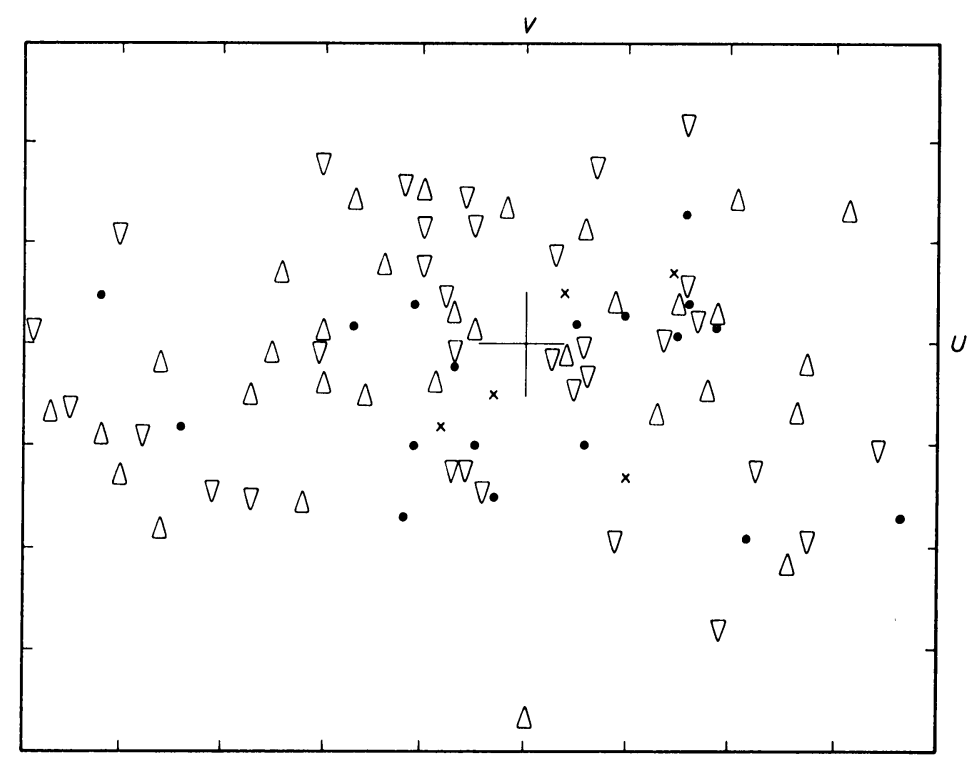

Fig. 3. Velocities of late type evolved stars: $F(\bigcirc), G(\triangle), K(\nabla), M(\times)$.

A numerical analysis can be made as follows. The quadratic $A U^{2}+2 F U V+B V^{2}$ can be referred to its principal axes so that it becomes $\mathfrak{A} u^{2}+\mathfrak{B} v^{2}$ by the substitution

$$
u=l U+m V \quad v=-m U+l V
$$

where $l=\cos \theta$ and $m=\sin \theta$ and

$$
\tan 2 \theta=-2 F / A-B \text {. }
$$

Now if the velocity function is elliptic, i.e.

$$
N(U, V)=\text { const } \times \exp \left\{-\left(A U^{2}+2 F U V+B V^{2}\right)\right\},
$$

the ratios of the mean values of $U^{2}, U V$ and $V^{2}$ are given by

$$
\overline{U^{2}}: \overline{U V}: \overline{V^{2}}:: B:-F: A,
$$

so that the deviation of the vertex $\theta$ can be found from

$$
\frac{1}{2} \tan 2 \theta=\begin{gathered}
\sum U V \\
\sum U^{2}-\sum V^{2} .
\end{gathered}
$$


Once $\theta$ has been found we can compute $l=\cos \theta$ and $m=\sin \theta$ and then find the ratio of the principal axes from

$$
\mathfrak{A} / \mathfrak{B}=\frac{\left(A l^{2}-B m^{2}\right)}{\left(B l^{2}-A m^{2}\right)}=\begin{aligned}
& \left(l^{2} \sum U^{2}-m^{2} \sum V^{2}\right) \\
& \left(l^{2} \sum V^{2}-m^{2} \sum U^{2}\right)
\end{aligned} .
$$

Applied to the stars selected for illustration the results are given in Table I.

TABLE I

\begin{tabular}{llll}
\hline Class of stars & No. of stars & Deviation & Ratio of principal axes \\
& & 0 & \\
& 66 & $+22^{\circ} .3$ & $(\mathfrak{A} / \mathfrak{B})^{1 / 2}$ \\
Sp. Type A & 33 & $+19^{\circ} .2$ & 2.85 \\
$\mathrm{Ca}^{+}$emission +3 & 65 & +3.0 & 1.41 \\
$\mathrm{Ca}^{+}$emission zero and minus & 69 & +3.1 & 1.82 \\
Late type evolved stars & & &
\end{tabular}

In all cases a ceiling has been imposed, namely only those stars for which the eccentricity of the galactic orbit does not exceed $e=0.15$ have been kept. This has been done partly to avoid loading the products $\sum U^{2}, \sum U V$ and $\sum V^{2}$ with very high entries and partly to avoid confusing changes in deviation due to stellar age with changes due to the eccentricity of the orbit - since the young stars are all stars with low eccentricity. A breakdown of the data for the old stars confirms the result that the low tilt is not a matter of eccentricity. Notice that the ratio of the principal axes is quite different for the young and old stars.

3. The initial conditions which give rise to the appearance of a particular velocity ellipse in the neighbourhood of the sun can be investigated with the help of Bok's (1934) Equations (1). They are of course very similar to equations used by Lindblad.

We consider motion in two dimensions only (that is, in the plane of the galaxy).

If $(x, y)$ are rectangular coordinates in fixed axis in the galaxy $(R+\xi, \eta)$ are rectangular coordinates in axes rotating with a constant angular speed as in the galaxy; then at an instant when the $(R+\xi)$ axis makes an angle $\theta$ with the $x$-axis

$$
\begin{aligned}
x= & (R+\xi) \cos \theta-\eta \sin \theta & y= & (R+\xi) \sin \theta+\eta \cos \theta \\
\dot{x}= & (\dot{\xi}-\eta \omega) \cos \theta & \dot{y}= & (\dot{\eta}+(R+\xi) \omega) \cos \theta \\
& -(\dot{\eta}+(\xi+R) \omega) \sin \theta & & +(\dot{\xi}-\eta \omega) \sin \theta \\
\ddot{x}= & \left(\ddot{\xi}-2 \dot{\eta} \omega-(\xi+R) \omega^{2}\right) \cos \theta & \ddot{y}= & \left(\ddot{\eta}+2 \dot{\xi} \omega-\eta \omega^{2}\right) \cos \theta \\
& -\left(\ddot{\eta}+2 \omega \dot{\xi}-\eta \omega^{2}\right) \sin \theta & & +\left(\xi-2 \eta \omega-(R+\xi) \omega^{2}\right) \sin \theta
\end{aligned}
$$

and when $\cos \theta=1$ and $\sin \theta=0$ (i.e. in axes rotating with angular velocity $\omega$ )

$$
\begin{aligned}
x & =R+\xi & y & =\eta \\
\dot{x} & =\dot{\xi}-\eta \omega & \dot{y} & =\dot{\eta}+(R+\xi) \omega \\
\ddot{x} & =\ddot{\xi}-2 \dot{\eta} \omega-(R+\xi) \omega^{2} & \ddot{y} & =\ddot{\eta}+2 \dot{\xi} \omega-\eta \omega^{2} .
\end{aligned}
$$


If $r$ is the distance from the centre of the galaxy, or $r^{2}=x^{2}+y^{2}=(R+\xi)^{2}+\eta^{2}$, the forces $F(x)$ and $F(y)$ directed to the centre of the galaxy are related to the force $F(r)$ by

$$
F(x)=\frac{R+\xi}{x} F(r), \quad F(y)={ }_{r}^{n} F(r)
$$

and if we approximate to the galactic attraction by setting

$$
F(r)=-\alpha r^{-2}-\beta r^{-3},
$$

then, ignoring quantities of the second order,

Now

$$
\begin{aligned}
& F(x)=-\alpha R^{-2}-\beta R^{-3}+\xi\left(2 \alpha R^{-3}+3 \beta R^{-4}\right) \\
& F(y)=-\eta\left(\alpha R^{-3}+\beta R^{-4}\right)
\end{aligned}
$$

$$
\ddot{x}=F(x) \text { and } \ddot{y}=F(y) .
$$

Choose $\omega$ so that

Then

$$
R \omega^{2}=\alpha R^{-2}+\beta R^{-3}
$$

$$
\begin{aligned}
& \ddot{\xi}-2 \dot{\eta} \omega-\xi\left(3 \omega^{2}+\beta R^{-4}\right)=0 \\
& \ddot{\eta}+2 \dot{\xi} \omega=0 .
\end{aligned}
$$

These are Bok's equations for the case $F(r)=\alpha r^{-2}+\beta r^{-3}$. These equations admit of a solution, namely if

$$
\xi=A \cos (p t+\gamma)+\xi_{0}, \quad \eta=-B \sin (p t+\gamma)+\hat{\lambda} t+\eta_{0}
$$

$$
\begin{aligned}
& -p^{2} A+2 \omega p B-A\left(3 \omega^{2}+\beta R^{-4}\right)=0 \\
& 2 \omega \lambda+\xi_{0}\left(3 \omega^{2}+\beta R^{-4}\right)=0 \\
& p B-2 \omega A=0 .
\end{aligned}
$$

Eliminating $A / B$ from (4) and (6) we have

$$
p^{2}=\omega^{2}-\beta R^{-4} \text {. }
$$

Given $R, \omega$ is fixed by (1) so that $p$ is fixed by (7). Since $A / B$ is fixed by (6) and $\lambda / \xi_{0}$ by (5), we are left with four disposable constants

$$
A, \gamma, \xi_{0}, \eta_{0}
$$

to be determined by the initial conditions, i.e. the values of $\xi, \eta, \dot{\xi}, \dot{\eta}$ at $t=0$.

4. Consider a set of stars at $\xi=0$ and $\eta=0$ at $t=0$ (i.e. the stars now in the solar neighbourhood) and consider a set of these whose velocities lie on an ellipse (centred on the circular velocity). If $\xi=0$ and $\eta=0$ at $t=0$,

$$
\begin{aligned}
& 0=A \cos \gamma+\xi_{0}, \quad 0=-B \sin \gamma+\eta_{0}, \\
& \dot{\xi}_{0}=-p A \sin \gamma, \quad \dot{\eta}_{0}=-p B \cos \gamma+\lambda .
\end{aligned}
$$


Hence

$$
\begin{aligned}
& \dot{\xi}_{0}=-\frac{p^{2}}{2 \omega} \eta_{0}, \\
& \dot{\eta}_{0}=\xi_{0}\left(\frac{p B}{A}+\frac{\lambda}{\xi_{0}}\right),
\end{aligned}
$$

using Equations (5) and (7). Hence if the velocities $\dot{\xi}_{0}$ and $\dot{\eta}_{0}$ lie on an ellipse so do $\xi_{0}$ and $\eta_{0}$ (which are in fact the coordinates of the epicentre relative to the local standard of rest). Let this ellipse be

$$
a \xi_{0}^{2}+2 f \xi_{0} \eta_{0}+b \eta_{0}^{2}=c^{2}
$$

But

$$
\begin{aligned}
& \xi=\xi_{0}+A \cos (p t+\gamma)=\xi_{0}(1-\cos p t)-{ }_{B}^{A} \eta_{0} \sin p t \\
& \eta=\eta_{0}+\lambda t-B \sin (p t+\gamma)=\eta_{0}(1-\cos p t)+\xi_{0}\left(\begin{array}{l}
\lambda t \\
\xi_{0}
\end{array}+\frac{B}{A} \sin p t\right),
\end{aligned}
$$

so that $\xi, \eta$ are given by the relations

$$
\begin{aligned}
& \xi=h \xi_{0}+k \eta_{0}, \\
& \eta=l \xi_{0}+h \eta_{0},
\end{aligned}
$$

where at any given time $h, l$ and $k$ are constants given by

$$
h=1-\cos p t, \quad k=-\frac{p}{2 \omega} \sin p t, \quad l=\frac{\lambda}{\xi_{0}} t+{ }_{p}^{2 \omega} \sin p t .
$$

Solving for $\xi_{0}, \eta_{0}$ in terms of $\xi$ and $\eta$, we obtain

$$
\begin{aligned}
\xi_{0} & =(h \xi-k \eta) /\left(h^{2}-k l\right) \\
\eta_{0} & =(h \eta-l \xi) /\left(h^{2}-k l\right) .
\end{aligned}
$$

Accordingly, if $\xi_{0}, \eta_{0}$ satisfy (8), $\xi, \eta$ satisfy

$$
a(h \xi-k \eta)^{2}+2 f(h \xi-k \eta)(h \eta-l \xi)+b(h \eta-l \xi)^{2}=c^{2}\left(h^{2}-k l\right)^{2}
$$

which can be written as

where

$$
\mathfrak{U} \xi^{2}+2 \mathfrak{F} \xi \eta+\mathfrak{B} \eta^{2}=c^{2}\left(h^{2}-k l\right)^{2},
$$

$$
\begin{aligned}
& \mathfrak{A}=a h^{2}-2 f h l+b l^{2}, \quad \mathfrak{B}=a k^{2}-2 f k h+b h^{2}, \\
& 2 \mathfrak{F}=-2 a h k+2 f\left(h^{2}+k l\right)-2 b h l .
\end{aligned}
$$

Accordingly $(\xi, \eta)$ lies on an ellipse also centred on the local standard or rest and given by (9). It also follows that every star whose velocity at $t=0$ lies within the original velocity ellipse, so that its epicentre $\left(\xi_{0}, \eta_{0}\right)$ lies within (8), lies inside the ellipse (9) at any time $t$. (It lies on an ellipse similar to (9) but with a smaller value of $c^{2}$.) 
5. It is convenient to work in units in which $R=1$ and $\omega=1$. These imply $\alpha+\beta=1$. In these units

$$
p^{2}=\alpha, \quad A / B=\sqrt{ } \alpha / 2, \quad 12 \lambda=-\xi_{0}(3+\beta) .
$$

In other investigations currently being pursued at the Royal Greenwich Observatory we use $\alpha=1.76245$. With this value of $\alpha$,

$$
\begin{aligned}
& p=1.32757, \\
& B / A=1.50651, \\
& \lambda=-1.11878 \xi_{0}, \\
& \dot{\xi}_{0}=-1.88122 \eta_{0}, \\
& \dot{\eta}_{0}=+0.88122 \xi_{0} .
\end{aligned}
$$

The ellipse which contains most of the velocities of the A-type stars and young $\mathrm{K}$ stars (with strong $\mathrm{Ca}^{+}$reversals) is

$$
2.297 U^{2}+6.320 U V+8.704 V^{2}=900\left(\mathrm{~km} \mathrm{~s}^{-1}\right)^{2}
$$

with the convention that $U$ is directed away from the centre of the galaxy, i.e. $U=+\dot{\xi}$; and $V$ is positive in the direction in which rotation takes place. Then

$$
8.704 \xi_{0}^{2}-6.320 \xi_{0} \eta_{0}+2.296 \eta_{0}^{2}=0.018544
$$

in the figure describing the limiting epicentres. At $t=-3$ and $t=-4$ we have the following limiting ellipse:

$\begin{array}{lcc} & t=-3 & t=-4 \\ \mathfrak{U} & 117.2 & 92.6 \\ 2 \mathfrak{F} & -23.46 & +11.32 \\ \mathfrak{B} & 3.296 & 1.54 \\ c^{2} /\left(h^{2}-k l\right)^{2} & 0.4625 & 0.2055 \\ \text { Tilt } & -5^{\circ} .8 & +3^{\circ} .5 \\ \text { Ratio of principal axes } & 7.49 & 7.76 \\ \text { Length of semi-axis major } & 0.47 & 0.41\end{array}$

At $p t=-2 \pi$, or $t=-4.74$, the ellipse degenerates to a straight line along the $\eta$-axis whose half length is 0.244 units.

6. For ellipses as large as this it is not really accurate to ignore the square of the eccentricity, which is implied in using the Bok equations; but the errors involved can be ascertained in any particular case by running a computer programme. The analytical and approximate solution gives a qualitative idea of the events; and the young stars now seen in the solar neighbourhood must have occupied areas very like the ellipses given by the approximate theory.

To see the significance of these areas, we enquire what are the figures corresponding 
to those velocities which we do not see in the young stars in the solar neighbourhood, and we examine the velocity ellipse.

$$
2.296 U^{2}+6.320 U V+8.704 V^{2}=900\left(\mathrm{~km} \mathrm{~s}^{-1}\right)^{2},
$$

that is an ellipse similar to (10) but tilted in the opposite sense.

The ellipse for $t=-3$ corresponding to (12) is

$$
22.85 \xi^{2}-16.38 \xi \eta+13.27 \eta^{2}=0.4625
$$

and it is shown in Figure 4 plotted together with the ellipse

$$
117.2 \xi^{2}-23.46 \xi \eta+3.30 \eta^{2}=0.4625
$$

which corresponds to (10). The shaded area is forbidden in the sense that if any stars had been found at $t=-3$ in this area, and had got to the solar neighbourhood at $t=0$, then their velocities would have been outside (10) (but inside (13) instead). The semi-axis minor of (14) is 0.063 units or 630 parsec. This means that the star forming arm had a width of 1260 parsec, which seems reasonable.

A direct computation was made of the positions of each of the stars shown in Figure 1, at $t=-4$, assuming only the force field

$$
F(R)=-1.76245 R^{-2}+0.76245 R^{-3}
$$

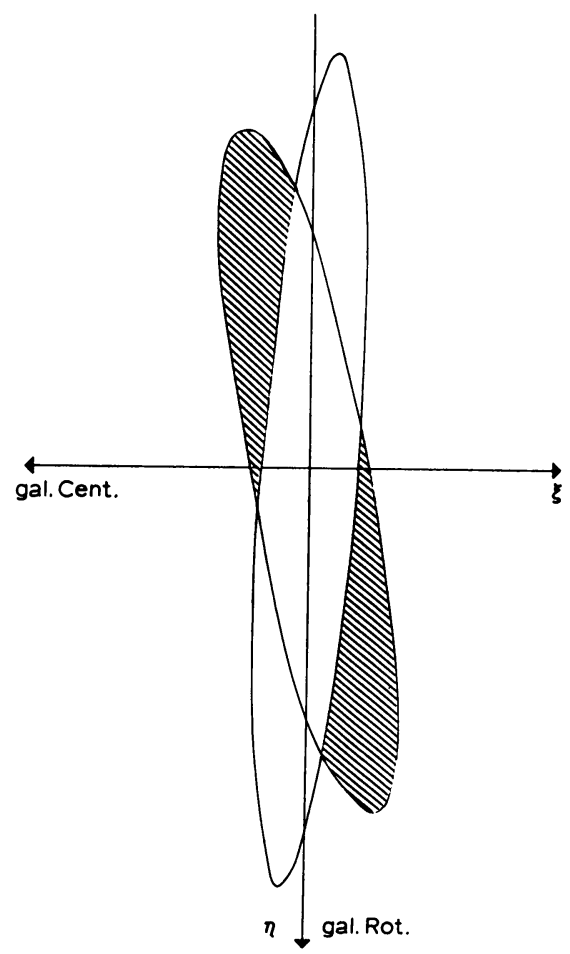

Fig. 4. Space distribution of stars, at $t=-4$, whose present position is the solar neighbourhood and whose velocities lie inside ellipses tilted at $+22^{\circ}$ and $-22^{\circ}$ (shaded). 
and the points are shown in Figure 5, in axes fixed in the galaxy at $t=-4$ and the origin being the position of the present centre of rest carried back to that time - i.e. the point occupied at $t=-4$ by an object now in the solar neighbourhood and having the circular velocity, or $U=0$ and $V=0$. This figure shows the points occupied in space, at that time, of objects which are all now in the solar neighbourhood, but are distributed in velocity space as we see in Figure 1.

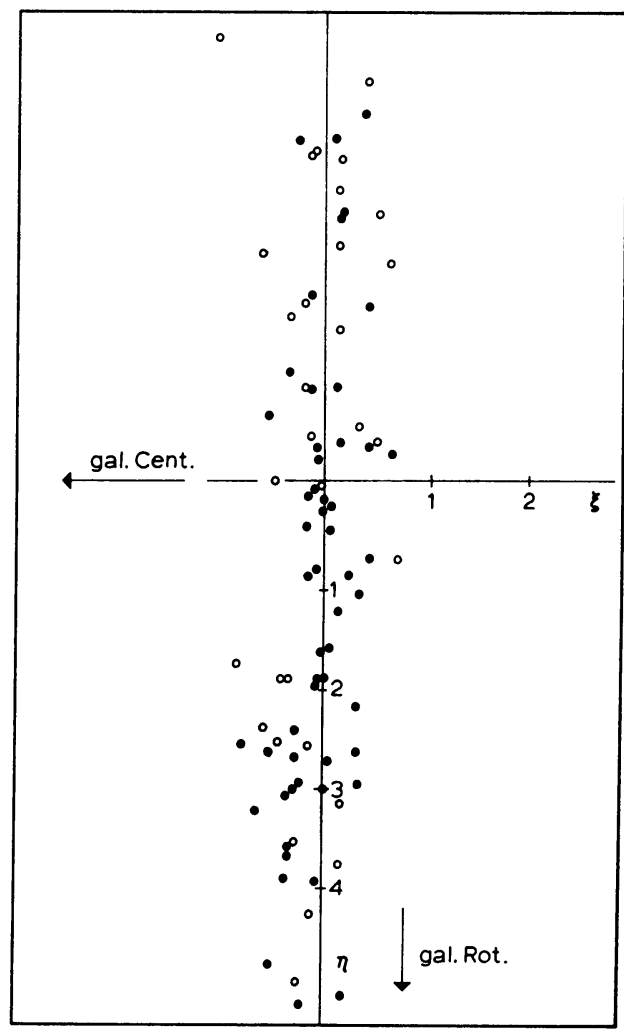

Fig. 5. Actual points in space, at $t=-4$, of the stars now in the solar neighbourhood having velocities (relative to the circular velocity) shown in Figure 1.

7. Figure 5 suggests that the young Gliese stars were formed in a region resembling a spiral arm at $t=-3$ or $t=-4$, or that is to say between 1.0 and $1.3 \times 10^{8}$ years ago. We may test this by enquiring how the stars compare with those in the Pleiades and Hyades clusters, whose ages Sandage (1957) placed at $2 \times 10^{7}$ and $4 \times 10^{8}$ years respectively.

This could be readily examined if we were able to draw a satisfactory HR diagram for the Gliese A stars. Unfortunately the only means of doing this is by constructing absolute magnitudes of the Gliese stars from their trigonometrical parallaxes, some of which are poorly determined, and it seems better to refer to spectral types, of which the counts are given in Table II 
TABLE II

Number of stars of a given spectral type

\begin{tabular}{llllllrlllll}
\hline Sp. & Pl. & Gl. & Hy & Sp & Pl. & Gl. & Hy & Sp & Pl. & Gl. & Hy \\
\hline B5 & - & 1 & - & A0 & 1 & 13 & - & A5 & 2 & 4 & 3 \\
B6 & 3 & - & - & A1 & 4 & 2 & - & A6 & 2 & 1 & 3 \\
B7 & 3 & 1 & - & A2 & 1 & 12 & 1 & A7 & 2 & 10 & 3 \\
B8 & 5 & 1 & - & A3 & 4 & 8 & 0 & A8 & 1 & 0 & 3 \\
B9 & 3 & 1 & - & A4 & 2 & 8 & 0 & A9 & $?$ & 2 & 4
\end{tabular}

Pl. = Pleiades $; \quad$ Gl. = Gliese $; \quad$ Hy = Hyades

These figures indicate strongly that the Gliese stars are much younger than the Hyades, and support the age of 1 to $1.5 \times 10^{8}$ years for the majority of the Gliese stars.

\section{Acknowledgement}

I am particularly indebted to Miss Rosalind Johnston, who put the programme described through the computer, and who has read and discussed with me the manuscript of this paper.

\section{References}

Bok, B. J.: 1934, Harvard Circular, No. 384.

Gliese, A.: 1957, Astron. Rechen-Inst. Heidelberg, Mitt. A, No. 8.

Gliese, A.: 1969, Veröff. Astron. Rechen-Inst. Heidelberg, No. 22.

Sandage, A.: 1957, in Proc. Vatican Conference on Stellar Populations, p. 41.

Strömberg, G.: 1946, Astrophys. J. 104, 12. 